\section{Cannabidiol and Its Impact on Depression}

\author{
Yarar $E^{*}$ \\ Co president of Nutrigenetic and Epigenetic Society, Istanbul, Turkey
}

\begin{abstract}
The endocannabinoid system (ECS) is a neuromodulator system with a crucial role in CNS and the reaction to endogenous and exogenous compounds and inflammation. Cannabidiol (CBD) is a basic part of ECS which is the overwhelming causative and/ or protective factor of Major Depressive Disease (MDD). CBD interacts with brain derived neurotrophic factor (BDNF) responds to inflammation, to dysregulations of hypothalamic-pituitaryadrenal (HPA) axis and many more imbalances in MDD patients for which ECS is a vital part to analyze, diagnose and reflect the treatment. ECS and the MDD appear to have strong connections and interactions and so interest in ECS and CBD use in MDD patients is developing as a rescue resort.
\end{abstract}

Keywords: Brain derived neurotrophic factor; Major depressive disease; natural compounds

\section{Introduction}

The pathophysiology of MDD has been increasing clearly following the development of neuroscience and bioinformatics. It mainly involves these aspects: The dysfunction of the brain related hormones, the hypothalamus-pituitary-adrenal (HPA) axis, the immune system, and the gut-brain axis (this latter parameter will not be dealt with in this review). It has obvious biological foundations, such as brain changes including unbalanced neurotransmitters, impaired neurogenesis, neuroplasticity decline, and abnormal neuronal circuitry. The most disturbing factor in MDD is it may or may not present relapses and/or remissions by several internal and external causes. Techniques and drugs come up short and so making the CBD an attractive and comfortable tool for acute and

*Corresponding author: Yarar E, Co president of Nutrigenetic and Epigenetic Society, Istanbul, Turkey Tel: +00 905313066556; E-mail: eyarar7@gmail.com

Citation: Yarar E (2020) Cannabidiol and Its Impact on Depression. J Transl Sci Res 3: 014.

Received: September 10, 2020; Accepted: September 29, 2020; Published: October 06, 2020

Copyright: () 2020 Yarar E. This is an open-access article distributed under the terms of the Creative Commons Attribution License, which permits unrestricted use, distribution, and reproduction in any medium, provided the original author and source are credited. chronic treatment options. To overcome the grave consequences many diverse targets should be considered such as mood enhancing catecholamines which bring about progressively positive state of mind and antidepressants work to build them to a satisfying level but cannot succeed in preserving that level in long term after cessation and cause many side and adverse effects accompany such as bone fractures, hyperlipidemia, suppression of bone marrow and endocrinological, cardiological, gastrointestinal issues mentioning only a few whereas CBD as observed balances catecholamines, acts on HPA axis dysregulation, reduces inflammations, regulates synaptic neuroplasticity and impacts almost on all systems in the body in a positive manner making it attractive as a main and/or an adjuvant treatment option. Role and function of endocannabinoid system and cannabidiol in MDD and endocannabinoid system's place in a brain of a person diagnosed with MDD should be brought to the attention of the academic circles. This mini review aims this and proposes that ECS might open a new avenue to form a unifying theory for MDD in future studies. MDD by large is still an obscure phenomenon and there are several theories in pursue to explaining it and this by isolating other psychiatric diseases. Often these theories come up also short in identifying the role of CBD's role in these theories. ECS's place in MDD is not sufficiently explored in full extent in academic circles but powerful indications show it might play a vital role in foreseeable future such as putting a unifying theory as ECS is interconnected with almost all cell communications in body and brain and MDD related regions. This is of utmost importance because due adverse effects and insufficiency of conventional psychiatric drugs in treating MDD which also posit numerous obscure parameters and so making CBD with its unique safety a reliable option. This review will look at the pathophysiological aspects of MDD, CBD's effect on MDD and ECS's impact in these parameters. Cannabidiols benefits aren't ignorable for diverse diseases arising from MDD. It seems a promising candidate as an adjuvant treatment option along with conventional medications for MDD. It has negligible side effects profile and pleiotropic benefits serving not only the "body" but also the "soul".

\section{Role and Function of Cannabidiol in MDD}

The essential components of the endocannabinoids are THC (tetrahydrocannabinol, a crystalline compound that is the main active ingredient of cannabis; it is psychoactive and anxiogenic) and CBD (cannabidiol a naturally occurring component in the resinous flower of cannabis; it is antipsychotic and anxiolytic). Although a balanced ratio of $\mathrm{CBD} / \mathrm{THC}$ brings also benefits in MDD this review focuses on CBD. ECS is composed of the cannabinoid receptors CB1 and $\mathrm{CB} 2$, the $\mathrm{CB} 1$ and $\mathrm{CB} 2$ endogenous ligands, AEA (N-arachidonoylethanolamine, AEA) and 2-arachidonylglycerol (2-AG) ,a specific and not yet identified cellular uptake mechanism and, the enzymes for endocannabinoid biosynthesis: $\mathrm{N}$ acyl phosphatidylethanolamine selective phosphodiesterase or glycerophosphodiesterase E1 and diacylglycerol lipase a or $\beta$, or inactivation: fatty acid amide hydrolase (FAAH) and monoacylglycerol lipase (MAGL) respectively for AEA and 2-AG. The ECS components arachidonate-based ECs, AEA 
(bliss molecule), 2-arachidonoylgycerol (2-AG) play positive roles in MDD whereas FAAH which clears AEA and 2-AG is a negative player and inhibition of FAAH is a vital part of treating MDD which is not a fact with standard medications and makes CBD's effect clear and indispensable. Cannabinoid receptors and their endogenous ligands are found all through the limbic system, where they modulate synaptic neurotransmission and influence MDD in a positive manner. A reduced ECS signaling might increment MDD side effects so improving the signaling appears to be a novel alternative for the treatment of MDD. CBD promotes antidepressant-like effects by enhancing the monoaminergic neurotransmission, inducing hippo multiple neurogenesis and normalizing the hyperactivity of hypothalamic-pituitary-adrenal (HPA) axis like antidepressants and so lessening and eventually eliminating MDD to an obvious extent.

ECS is a complex system with additional components such as putative CB1 antagonist peptides like hemopressins, peroxisome proliferator-activated receptor-a (PPAR-a) and its ligands, such as oleoylethanolamide (OEA) or palmitoylethanolamide (PEA), and $\mathrm{N}$-arachidonoyl-DA (NADA), which activates both TRPV1 and CB1 receptors. Memory function is mainly related to hippocampus, motor to basal ganglia, and mood to prefrontal cortex and so any dysfunction of the ECS can be considered as a causative factor in MDD. The CB1 receptor is one of the foremost abundant $\mathrm{G}$ protein-coupled receptors (GPCRs) within the CNS and is found in especially high levels within the neocortex, hippocampus, basal ganglia, cerebellum and brainstem CB1 receptors are moreover found on peripheral nerve terminals and a few extra-neural regions such as the testis, eye, vascular endothelium and spleen [1].

CB1 receptors are exceedingly improved at presynaptic and axonal compartments, confining their work to sites of synaptic action. In expansion to its area on the cell surface, intracellular localization of CB1 receptors has moreover been detailed in heterologous systems. CB2 receptors are expressed at much lower levels in the CNS compared to $\mathrm{CB} 1$. CBD indirectly affects the $\mathrm{CB} 1$ receptors by pausing the enzymatic breakdown of AEA, enhancing AEA, permitting it to remain in the system longer and provide positive medical benefits in MDD. Any dysfunction in this system leads not only to MDD but also to other psychiatric disorders and causes also some pathological issues which is not a subject to discuss in this review. Endocannabinoid signaling is widely distributed throughout corticolimbic circuits that are linked to the stress response. ECs are released from lipid precursors when needed and serve as reverse signaling messengers in GABAergic and glutamatergic synapses and modulators of postsynaptic transmission, interacting with other neurotransmitters including DA to which docking CBD has only minimal effect which is a negative fact for monoamines.

AEA can be characterized as a stress-responsive neurotransmitter, and is to a great extent not as tonically dynamic as 2-AG within the adult brain. Be that as it may, in the event that AEA is initiated to be always dynamic, the brain will downregulate its responsiveness to AEA, in this way diminishing its neuromodulatory usefulness, and the signaling work carried out by stress-responsive varieties of AEA levels will be disturbed meaning progression of MDD symptoms. AEA and 2-arachidonoylglycerol bind to a component of G-proteincoupled receptors, of which the cannabinoid CB1 receptor is deeply distributed in areas of the brain in charge of motor control, cognition, emotional responses, motivated behavior and homeostasis. 2-AG and AEA are synthesized and degraded by FAAH and CBD inhibits FAAH, thus increasing the AEA. Particular enzymatic pathways such as FAAH and so have physiological and pathophysiological parts to these two ECs by expanding or halting the MDD indications and preventing of emerging of it as a disease. AEA is synthesized within the brain, where levels are controlled by firmly directed degradation pathways. Its impacts within the brain are greatly localized. Like $2-\mathrm{AG}$, it is a retrograde neurotransmitter whose release can influence numerous cells within the neighboring synaptic space, but not past that space. It does not have the diffuse activity of a hormone; its release is locally controlled and does not influence the organism systemically within the way that exogenous cannabinoid organization does. AEA protects neurons.

AEA reestablishes the raised $\mathrm{CB} 1$ expression conjointly to promote the diminished level of 5-HT within the within the hippocampus. In expansion, AEA reestablishes the elevated NA levels within the prefrontal cortex and actuates a neuroprotective impact by reestablishing the diminished glutathione and expanded lipid hydroperoxides levels together with the diminished superoxide dismutase movement watched in hippocampus and/or prefrontal cortex which zone significant brain regions included within the control of temperament, aggression and/or impulsivity and decision making, which are unquestionably changed in MDD and all these demonstrate reversed MDD indications.

CBD is responsible for a sharp inhibition of neuronal activity, which is required to maintain complex firing patterns across widespread brain networks. Having such a fundamental role, it is no surprise that malfunctions in this system are associated with a myriad of brain disorders such as MDD including anxiety. CBD treatment interacts in a positive manner with AEA. Outside the brain, the EC is one of the critical modulators of the autonomic nervous system, the immune system and microcirculation when dysregulated causing MDD symptoms. Altered EC function causes pathophysiological aspects of MDD and related suicidal behavior [2] CB1 and CB2 are transmembrane G-protein coupled receptors (GPCRs) located in the central and peripheral nervous system. The most abundant and causative cannabinoid receptor is the CB1 cannabinoid receptor, but CB2 cannabinoid receptors, transient receptor potential (TRP) channels, and peroxisome proliferator activated receptors (PPAR's) are also involved by some cannabinoids.

As exogenous cannabinoid CBD interacts with 2-arachidonoyl glycerol (2-AG) and AEA and bring out its effects in MDD. MDD is also a disease of imbalances and dysregulation in structural and neuronal/nervous system. CBD inhibits endocannabinoid reuptake, activates transient receptor potential vanilloid 1 and $\mathrm{G}$ proteincoupled receptor 55 and enhances the activity of 5-HT1A receptors. As an essential part endogenous cannabinoid AEA acts as a partial agonist at $\mathrm{CB} 1$ receptors. $\mathrm{CBD}$ acts as an agonist at 5-HT1 A receptors [3] and so works as a fast-acting antidepressant and anxiolytic medication [4] A reduced affinity of 5-HT1A receptors is identified in the brains of MDD individuals. As a 5-HT1A serotonergic agonist and activator of endogenous endocannabinoid system CBD is an antagonist at GPR55 receptors, and an activator of transient receptor potential channels. CBD's activity at 5-HT1A receptors seems to drive its neuroprotective, antidepressive, and anxiolytic benefits, although the mechanism of action how CBD diminishes and eliminates the anxiety is still unclear. This is imperative as these 
receptors help in releasing brain derived neurotrophic factor (BDNF) and are responsible for neuroprotective impacts of the 5-HT receptors and these exceptionally receptors are too related to the neurobiology and etiology of MDD. The mechanisms how 5-HT1 A receptors adjust to stress and initiate antidepressant-like impacts are also not clear. Stressful events and insults impede post-synaptic 5-HT1A-mediated effects in limbic regions, in hippocampus, and cause individuals incapacity to adjust themselves to stress-induced behavioral results all aroused by MDD so bringing out to facit circles. The brain-derived neurotrophic factor (BDNF) is a protein located and manufactured primarily within the brain and spinal cord which fortifies the vitality of nerve cells and induces the growth, maturation and maintenance of them. It is active at synapses where cell-to-cell communication occurs which adjusts in reaction to events, a characteristic called Synaptic Plasticity (SP) so any impairments in SP apparatus contribute to psychiatric disorders. BDNF protein helps regulate SP. Evidence demonstrates that BDNF levels are decreased in MDD. Increased levels of BDNF seems to contribute to improving MDD symptoms. CBD brings BDNF to a desirable level intensely and keeps this adjust in long term application by progressing and treating MDD symptoms.

CBD invigorates in acute stages fast and in chronic stages maintained antidepressant-like impact relevant for MDD. These impacts is related to swift changes in SP within the medial prefrontal cortex through activation of the BDNF-TrkB signaling pathway. CBD can also act specifically on GABA receptors which is one of the essential neurotransmitters of the brain related to MDD. When a neuron is under extraordinary inhibition due to gamma aminobutyric acid (GABA) action, it can synthesize 2-AG and AEA and send them to the previous neuron whereupon they dock to $\mathrm{CB} 1$ and $\mathrm{CB} 2$ receptors, driving to a cascade of molecular responses that eventually diminishes GABA release. The diverse capacities of GABA are determined by the area of its receptors as well as their particular structure. Each GABA receptor is comprised of 5 proteins drawn from a pool of 19 potential proteins, with diverse arrangements determining what substances can enact them and how they work with respect to potency, speed and duration of activity. Recently, it has been found that the endocannabinoids (ECs) can too act specifically on GABA receptors in a way that's exceptionally similar to how benzodiazepines barbiturates etc drugs work. It is well possible that such a direct modulation of GABA receptors contributes to the restorative impacts of CBD observed in MDD. CBD and related compounds including particular terpenes offer promising treatment options for MDD. Prove of the part of the ECS within the neurobiology of neuropsychiatric disorders is starting to rise. The behavioral impacts of ECs are mediated through the central cannabinoid $\mathrm{CB} 1$ receptor. Imbalanced ECs, and within the density and coupling efficacy of $\mathrm{CB} 1$ receptors, have been reported within the prefrontal cortex of MDD patients. ECS is a ubiquitous lipid signaling system and has vital administrative capacities all through the body including innate and immune system. According to studies forebrain 5HT1A receptors are primary targets of antidepressant drugs. The mechanism is still open to debate, but these receptors interfere in neuroplastic changes driving to neurogenesis Antidepressant treatment may increase hippocampal neurogenesis but once more with undesirable negative impacts where CBD appears its effect with nearly null side impact. CBD's antidepressant impacts mediated by help of 5-HT1A receptor-mediated neurotransmission and its anxiolytic activity in connection to MDD in details are yet to be decided.

\section{Cannabidiol's Place in MDD and Related Brain Regions}

The prefrontal cortex, hippocampus, and amygdala play an imperative role within the control of emotion, stress reactions, selfcontrol, cognitive response, but in MDD patients the function of the prefrontal cortex and hippocampus are impaired, whereas the movement of the amygdala is expanded. In this manner, long-lasting antidepressant treatments point to extend the neurotrophic variables, fortify neurogenesis, diminish the hippocampus neuronal apoptosis, and progress the mood and cognition however none of them suffice to prevent the relapses and amplifying the remission periods of MDD which with side impacts; in this manner CBD's impact with neglectable side effects is shown multifaceted and with self-evident success. Ecb's such as AEA (N-arachidonoyl-ethanolamine, AEA) and 2-arachidonylglycerol (2-AG) act by activating cannabinoid type 1 (CB1) or 2 (CB2) receptors. The anxiolytic effect of medications such as CBD which initiate Ecb's impacts on increase in AEA levels in several encephalic regions, including the prefrontal cortex (PFC) and so activation of Cannabidiol 1 (CB1) receptors by $\mathrm{CB} 1$ agonists infused direct into these regions is usually anxiolytic.

In any case, depending on the encephalic locale being investigated on the stressful events, reverse impacts are observed, as detailed interior the ventral hippocampus which relates to stress, feeling and influence. Anxiolytic impacts of CBD in models of MDD are associated to specific receptor components and brain districts. The midbrain dorsal periaqueductal gray is integral to anxiety, organizing autonomic and behavioral reactions to threat and periaqueductal gray (PAG) prompting in humans produces thoughts of distress and fear. Just to name this region shows how deeply MDD affects a person's life because PAG is an anatomic and functional interface between the forebrain and the lower brainstem and incorporates a major part in integrated behavioral reactions to internal (e.g., torment) or external threat stressors. The PAG comprises of distinct columns that get specific inputs from the prefrontal cortex, amygdala, hypothalamus, and nociceptive pathways and any disorder facilitates symptoms of MDD. By means of its associations with diverse brainstem nuclei, the PAG facilitates particular patterns of cardiovascular, respiratory, motor, and pain modulatory reactions. These reactions shift according to the sort of stress and the subject's perception of the threatening stimulus which is positively altered by introduction of CBS.

With regard to fear CBD plays its part in different components of the brain. As well as the neural circuitry supporting fear memory handling comprises other inter-connected zones such as the hippocampus, amygdala, and PAG. Infusing CBD into the PAG diminishes anxiety in paradigms assessing intrinsic fear. In any case, this zone is additionally imperative for intervening freezing and other protective behaviors in reaction to learned threats, proposing that $\mathrm{CBD}$ regulation of fear memory expression may include the PAG. In humans CBD decreases anxiety and autonomic excitement amid the seeing of fearful facial expressions, which is accompanied by diminished activity in and functional connectivity between the amygdala and $\mathrm{mPFC}$. In mice CBD diminishes amygdala activation as measured by c-Fos expression. This raises the plausibility that the amygdala plays a part in mediating CBD control of fear memory. 
CBD also decreases mPFC-hippocampus functional connectivity amid cognitive processing. This proposes that the hippocampus may moreover be involved in CBD regulation of certain aspects of learned fear. After termination, fear expression is low when tried within the extinction setting but fear renewal happens exterior of this context. This relevant control of fear termination includes the hippocampus and its associations with the $\mathrm{mPFC}$ and amygdala. CBD might decrease fear relapse and/or the spontaneous recovery of fear that happens over time after termination through its activities on the hippocampusmPFC-amygdala circuit. CBD modulation of this circuitry may also regulate learned fear in other paradigms with translational significance. CBD moreover diminishes functional connectivity between the amygdala and anterior cingulate cortex ACC a brain region where both affective and cognitive information converge; pointing to both an anxiolytic effect of CBD and a critical modulatory role of the ACC which apart from the emotion-regulating properties of $\mathrm{CBD}$, the $\mathrm{CBD}-\mathrm{ACC}$ relationship has not been systematically investigated too. Endocannabinoid signaling is broadly dispersed all through corticolimbic circuits that are connected to the stress reaction. Distinctive scanning procedures show that AEA and 2-AG are synthesized within the hypothalamus, amygdala, hippocampus and prefrontal cortex Autoradiography of cannabinoid receptors in brain areas from a number of mammalian species, including human, reveals a unique and preserved conveyance; binding is most dense in surge nuclei of the basal ganglia--the substantia nigra pars reticulata and globus pallidus--and inside the hippocampus and cerebellum. The exact importance of these volumetric varieties from the norm is still obscure.

MDD appears to lie in various brain areas as well as no place in particular. A number of clarifications can be deciphered and have been proposed with regard to this state of affairs. One plausibility is that MDD is localized in numerous brain regions at the same time. This may emerge from a common molecular peculiarity inside the neurons of multiple brain regions. Receptor variations from the norm or varieties from the standard in signal transduction might be such conceivable anomalies. Conflicting results have been detailed after CB1 enactment inside the dorsal hippocampus (dHIP) which performs fundamentally cognitive functions however cortical brain regions included in MDD are the dorsal and medial prefrontal cortex, the dorsal and ventral front cingulate cortex, the orbital frontal cortex and the insula are moreover influenced. The main subcortical limbic brain districts involved in MDD are the amygdala, hippocampus, and the dorsomedial thalamus and gene expression in the dorsal hippocampus correlates with cortical regions involved in information processing, while genes expressed in the ventral hippocampus correlate with regions involved in emotion and stress (amygdala and hypothalamus) all docked to ECS and CBD acts on all of them via epigenetic regulation.

Both structural and functional anomalies in these regions have been found in MDD. Decreased hippocampal volumes and amygdala have been noted in subjects with MDD. CBD moreover attenuates bloodoxygen-level dependent activation within the left amygdala, and the front and posterior cingulate cortex in reaction to escalation frightful faces, and diminishes amplitude in skin conductance fluctuation, which is exceedingly connected with amygdala activation. Subjects who remit with treatment have indeed been appeared to have bigger pretreatment hippocampal volumes whereas those with smaller hippocampal volumes were reported to be more inclined to relapse.
Diminished amygdala core volume has been reported in MDD but $\mathrm{CBD}$ is shown to have no impact on gray matter volume and cortical thickness and short-term exposure to exceedingly purified CBD does not influence cortical macrostructure but reestablishes hippocampal and amgydala volume loss and in fact most MDD patients have diminished size of amygdala as shown in MRI. (Personal record) CBD decreases the reduced hippocampal neurogenesis and dendrite spines thickness initiated by consistent stress and avoids microglia activation and the diminish within the number of parvalbuminpositive GABA neurons MDD and equalizations cell destiny regulatory pathways such as autophagy and others essential pathways for neuronal survival and so advancing the obliterating results related with neurodegeneration [5] In spite of the fact that mood disorders are not typical neurodegenerative disease, neural progression, diminished neurogenesis and neuroplasticity, inflammation, cell death and dysregulation of monoamines occurs in a few patients with MDD.

An imbalance inside the fundamental excitatory (glutamate) and inhibitory (GABA) neurotransmitter systems inside the brain plays a part within the pathophysiology of MDD. Subsequently, the ECS appear to represent a therapeutic target for MDD as a regulator of glutamate and GABA release by means of the $\mathrm{CB} 1$ receptor (CB1R). CBD can reestablish cannabinoid/GABAergic signaling deficits in regions of the brain implicated in pathophysiology of MDD. Also, CBD increases mTOR signaling in rat models of neurodegeneration which is an additional burden on MDD patients. It is still obscure whether these instruments participate in CBD induced antidepressant effects. All these processes and effects are still open to talk about and require more studies.

\section{Theories on Pathophysiology of MDD and CBDs' Antidepressant Mechanism}

ECS is highly involved in MDD and CBD has several pleiotropic roles in all of them as regulating factor and explains in great extent the causes of MDD and the application of CBD affecting the ECS. There are some pathophysiological speculations how really MDD as a disease emerges. These theories are actually interconnected with an obvious need for a unifying theory. In fact the logical way is considering all of them in identifying the MDD. One by one and derived from different approaches these theories along with other minor ones come up very short in explaining the etiology of MDD but they may reflect diverse spectrums of MDD and it is worth to sum up them here with their relation to ECS and CBD.

\section{Monoamine Theory and Cannabidiol}

Monoamine theory depicts that MDD is an aftereffect of a distorted monoamine synapse framework, especially; serotonin (5HT), noradrenaline (NA), norepinephrine (NE) and dopamin (DA) lowering extracellular monoamines complexes and neurotransmission and suggests that the basis of depression is a reduction in the levels of 5-HT, DA, and NE in the body. ECS is highly interconnected with the monoamine neurotransmitters. In fact one study indicates that CBD reduces DA level without affecting hypothalamus, increases 5-HT level in hypothalamus stimulating neurotransmitter synthesis. CBD reduces hypothalamic NE synthesis and DA release, inhibits 5-HT synthesis so showing its anxiolytic role. One other study found out that CBD's anxiolytic role is dependent on brain 5-HT levels but not NA so CBD can exert its effect on cathecolamines indirectly it is clinically active only as a neglectable and partial agonist at the DA D2 receptors [6]. 
In addition to binding to $\mathrm{CB} 1$ and $\mathrm{CB} 2$ receptors of the endocannabinoid system, there's prove that CBD sanctions 5-HT1A serotonergic and TRPV1-2 vanilloid receptors, antagonizes alpha-1 adrenergic and $\mu$-opioid receptors, represses synaptosomal take-up of NA, DA, 5-HT and gammaminobutyric acid and cellular takeup of AEA, acts on mitochondria $\mathrm{Ca} 2$ stores, blocks low-voltageactivated (T-type) $\mathrm{Ca} 2$ channels, invigorates activity of the inhibitory glycine-receptor, and restrains activity of fatty acid amide hydrolase (FAAH) and BDNF-TrkB interactions may modulate catecholamine release from adrenal chromaffin cells under acute stress conditions. There are also evidences of CBD's modulatory effects on DA activity within the mesolimbic pathway, functional interactions with the 5-HT receptor system, and their downstream molecular signaling effects relieves the symptoms in MDD and the inhibitory action of CBD on catecholamine secretion at the level of the adrenal medulla is clearly observed with this context CBD improves and restores 5-HT and $\mathrm{BDNF}$ levels via $5 \mathrm{HT} 1 \mathrm{~A}$ receptor activation and restoring 5-HT levels. There are $\mathrm{CB} 1$ receptors located in hippocampus and amygdala and any stress and anxiety is a result of the bed nucleus of the stria terminalis coordinating a stress-based response in conjunction with the amygdala which affects catecholamines. CBD produces its anxiolytic effects via activation of the 5HT-1a receptor associated with 5-HT, affecting positively the amygdala. Studies show that CBD acts via the modulation of the 5-HT1A receptors.

The acute anxiolytic and antidepressive activities of intense CBD are proposed to be intervening by $5 \mathrm{HT} 1 \mathrm{~A}$ receptors. The crosstalk among cannabinoids and serotoninergic signaling is unpredictable. In rodents, $\mathrm{CBD}$ administration into the dorsal parcels of periaqueductal gray matter (dPAG) produces against aversive impacts in the elevated plus maze and flight-induced by local electric stimulation [7] other cerebrum regions such as the basal ganglia, the bed nucleus of stria terminallis, the prelimbic PFC [8] and the dorsal raphe core likewise appear to mediate $\mathrm{CBD}$ impacts through 5HT1A receptors.

The molecular mechanism by which CBD encourages 5HT1A receptor activation is still obscure. Evidence proposes that it might include allosteric regulation of this receptor, advancing 5HT1A agonist-related stimulation of [35S] GTPS binding increment in 5 -HT release or potentially reuptake inhibition or the circuitous development of heterodimers comprising of 5HT1A and different receptors, for example, $\mathrm{CB} 1$. NE plays a role in stress reactions and the control of feelings. NE levels have been found to be low in conditions of MDD. NE enacts the amygdala. The amygdala can too improve long-term capacity of stressful memories within the hippocampus and striatum.

Cannabinoids repress epinephrine (adrenaline) secretion in rabbit isolated adrenal organs. Since adrenaline in plasma starts solely from adrenal chromaffin cells, impacts of cannabinoids on adrenaline release were considered in isolated adrenal organs. In vivo, catecholamine exocytosis from chromaffin cells is controlled by the activity of presynaptic splanchnic nerves innervating the adrenal medulla. Incitement of the nerve terminals causes release of acetylcholine and non-cholinergic neurotransmitters (e.g., opioid peptides and vasoactive intestinal peptide), which at that point enact postsynaptic receptors to evoke catecholamine secretion. With regard to stress induced by epinephrine cannabinoids brings down the electrically evoked adrenaline release in rabbit isolated adrenal glands; the likely component could be a presynaptic CB1 receptor-mediated inhibition of acetylcholine release from preganglionic sympathetic neurons innervating the adrenal medulla. This diminishment in adrenaline release most likely accounts for the marked decrease in plasma adrenaline concentration and reduced stress observed after cannabinoid administration within the pithed rabbit [9] which offers $\mathrm{CBD}$ as an alternative to treat MDD patients suffering from extensive release of epinephrine causing stress with no motive either.

ECs and their receptors, basically the CB1 receptor type, work as a retrograde signaling system in numerous neural connections inside the CNS, especially in GABAergic and glutamatergic synapses. They moreover play a modulatory work on dopamine (DA) transmission, in spite of the fact that CB1 receptors don't show up to be found in Dopaminergic terminals, at least within the major brain districts receiving dopaminergic innervation, e.g., the caudateputamen and the nucleus accumbens/prefrontal cortex. In this manner, the impacts of cannabinoids on DA transmission and DA-related behaviors are by and large indirect and exerted through the balance of GABA and glutamate inputs received by dopaminergic neurons.

Although cannabinoid $\mathrm{CB} 1$ receptors (CB1Rs) are densely expressed in neurons expressing DA D1 receptors (D1Rs), it isn't completely understood to what degree they modulate emotional behaviors. Cannabinoid-induced increments in nucleus accumbens DA concentration are thought to emerge from an increase within the mean firing rate of DA neurons inside the ventral tegmental range. Dopaminergic neurons of the midbrain are the most source of DA (DA) inside the mammalian CNS. Their loss is related with one of the first recognizable human neurological disorders, Dopaminergic neurons are found within the substantia nigra pars compacta. In spite of the fact that their numbers are few, these dopaminergic neurons play a crucial role inside the control of various brain capacities including intentional movement and a wide cluster of behavioral forms such as mood and stress. Another point is that decision-making which is an ethologically adaptive component that's impeded in MDD. Action inside the mesocorticolimbic DA system has been traditionally related with decision-making.

The endocannabinoid system through its actions on inhibitory and excitatory synapses modulates DA activity and decision-making. Cannabinoid-DA interactions paying emphasis in two forms where DA has been proposed as a key neurotransmitter, such as the basal ganglia function and the corticolimbic processes. It is apparent that dysregulation or overactivity of DA transmission where CBD might be of therapeutic potential for MDD conceivably through activities that facilitate, among others, a normalization of DA transmission.

The diminishing in monoamine neurotransmission results either from too little synapses released by presynaptic neurons, or from quickly reabsorbed/manipulated synapses or a lessening in postsynaptic receptors but also observed in other psychiatric conditions not making it a sole player in MDD. Studies have conflicting results and despite any positive observations monoamine theory is still being very much open to debate.

\section{Dysregulation of Hypotalamic-Adrenal-Axis (Hpa Axis) Theory and Cannabidiol}

The HPA axis could be a crucial portion of the stress reaction system, and dysfunction of the HPA axis is one of 
the foremost imperative mechanisms behind MDD. Both mental and physiological stress enact the HPA axis and invigorate the release of corticotrophin-releasing figure (CRF) and vasopressin (AVP) by the hypothalamus. Both CRF and AVP start the anterior pituitary organ to release adrenocorticotrophic hormone (ACTH), which progresses the release of adrenocortical hormones, including glucocorticoid (GC), and causes an increase in circulatory GC levels, which controls the secretion of CRF and AVP by the hypothalamus, forming a negative feedback circuit.

In any case, over half of the MDD patients show negative feedback dysfunction of the HPA axis, including a constant increase in circulatory $\mathrm{GC}$ and $\mathrm{ACTH}$, and some of the patients in fact endure from hypercortisolemia. Cortisol is the stress hormone and it lies at the crossing point of our stress response and anxiety. Stress leads to damage or inhibition of neurogenesis through hypercortisolemia, reduced BDNF, or extended glutamate. Cortisol seems to form a domino effect that hardwires pathways between the hippocampus and the amygdala in a way that might make an appalling cycle by making a brain that gets to be inclined to be in a consistent state of fight-or-flight. In case of intense or unexpected stressful situations ECS works to counter the spike in cortisol in arrange to bring the system back to adjust. It is persistent cortisol and stress that definitely depletes this system. Chronically raised cortisol can cause brain damage. This damage can result in anxiety an additional negativity figure of MDD.

CBD diminishes cortisol levels, support stress response framework (AEA) and energizes brain repair by means of neurogenesis and this comes about in a coordinate impact on uneasiness both brief and long term. In spite of the exceptionally strong preclinical and clinical data of a dysregulation of the HPA axis in stress-related mental disorders, such as MDD, no drug has been approved that targets particular components of the HPA axis. In extension, no test is routinely utilized interior the clinical setting to recognize patients with a measureable HPA axis anomalies. In fact, there's prove that not all MDD patients show alterations of the HPA axis, and subsequently not all of them would benefit of a very particular treatment, centering on only HPA axis components. Major modifications of the HPAaxis system that can be exchanged by antidepressant treatment are regularly seen in MDD patients. Persuasive prove focuses to the incorporation of a dysfunctional Glucocorticoid Receptor (GR) system in these changes. Support for this also comes from studies of transgenic mice that express an antisense RNA, complementary to the GR mRNA, and have different neuroendocrine characteristics of human depression as well as altered behaviour. Several of these neuroendocrine and behavioral characteristics of the transgenic mice can be exchanged by antidepressants. A conceivable clarification for this may be that the antidepressant-induced increase in GRs renders the HPA axis touchier to glucocorticoid feedback. Glucocorticoid receptor (GR) plays an important role in the HPA axis function during depression; the excessive circulating GC reduces the sensitivity of GR, while antidepressant therapies increase the GR expression, enhance the GR function, and improve the negative feedback medicated by GR. Later research found that HPA axis dysfunction also reduces BDNF expression [10] inhibits 5-HT synthesis, decreases Glu receptor expression, and even disturbs neuroplasticity and neural circuits.

The amygdala invigorates the nerve center which thusly energizes the HPA axis which by expanding blood cortisol complexes follows up on a positive way component to influence the amygdala. Over-excitation of the HPA axis is prevented with a negative examination instrument by the hippocampus which limits the HPA axis action dysfunction. Studies show raised cortisol, ACTH and corticotrophin releasing hormone $\mathrm{CRH}$ levels in patients with MDD and raised glucocorticoid complexes appear to cause restraint of average prefrontal cortex excitation in MDD. Low blood BDNF levels are observed altogether in MDD. CBD controls acute stress HPA actuation impacting 5-HTR1A receptors. Constant stress and inflammation cause both the HPA axis and ECS dysfunction. It is critical to address the endocannabinoid deficiency and rebalance the HPA axis and increase the BDNF levels.

The ECS can be rectified by bringing down CRH and FAAH with exogenous $\mathrm{CBD}$, thereby raising AEA and lowering glutamate. Expanded levels of AEA are specifically correlated with improved wellness, mood, and stress reaction and so making CBD an excellent tool to boost endocannabinoid signaling, progress the regulation of the HPA axis. CBD actually increases ECS tone which helps also to improve the control of homeostasis over the HPA axis. It is critical to reestablish the endocannabinoid deficiency and rebalance the HPA axis to which CBD shows its positive impacts by raising AEA and bringing down glutamate. Expanded levels of AEA are specifically related with improved mood and so reducing negative shape of MDD by bringing down cortisol and CRH levels.

Diminishment of AEA-CB1R signaling inside the amygdala intervenes the anxiogenic impacts of $\mathrm{CRH}$, and $\mathrm{CB} 1 \mathrm{R}$ activation is fundamental to negative feedback of the neuroendocrine stress response, and secures against the opposing impacts of chronic stress; chronic stress disables eCB signaling inside the hippocampus and amygdala, driving to anxiety and individuals with MDD show up elevated $\mathrm{CB} 1 \mathrm{R}$ and diminished peripheral AEA, suggestive of diminished $\mathrm{eCB}$ tone. There are no critical connections between cortisol levels and sBDNF levels however low BDNF might contribute to the neurobiology of MDD. The ability of cannabidiol to regulate acute stress HPA activation might be explained, at least in part, by its action on 5-HTR1A receptors. Cannabidiol regulates the HPA axis responses to stress and impairment of eCB signaling in the hippocampus following acute/chronic stress could contribute to the development of MDD. Finding new eCBs molecular targets to modulate the "stressed hippocampus", therefore, could be a helpful contribution for novel therapeutic approaches yet there are no drugs available so far making CBD again a reliable medication. Although several studies indicate a strong connection in-between MDD and HPA axis dysfunctions this theory of HPA axis is also open to debate and cannot be considered as a sole contender.

\section{The Inflammatory Hypothesis of MDD and Cannabidiol}

One other essential approach to MDD is the inflammatory hypothesis which posits that immune hyper activation and dysregulated cytokine production are involved in MDD. Cytokines are cell signal transducing proteins or polypeptides that intervene and direct immune reactions and inflammation. They cross the bloodbrain barrier, in this manner forming numerous perspectives of MDD pathophysiology, including enteric and general neurotransmitter system, neuroendocrine function, and neural plasticity. Be that as it may, there's clear prove that changes in cytokine levels are not reliably related with positive results amid MDD treatment. 
Inflammatory hypothesis sets that in MDD the proinflammatory cytokines, including IL-6 and TNF-a, increase in sum whereas the anti-inflammatory cytokines, including interleukin-10 (IL-10) and transforming growth factor-beta (TGF- $\beta$ ), diminish, making the holistic resistant response tend to inflammation. The excessive proinflammatory cytokines repress the negative feedback of the HPA axis, increase the permeability of the blood-brain barrier, decrease the synthesis of 5-HT, disturb the glutamatergic frameworks, and result in depression.

The proinflammatory cytokines, in expansion to actuating the hypothalamic-pituitary-adrenal axis and in this manner expanding cortisol synthesis, also enact the the tryptophan-kynurenine pathway. This comes about within the synthesis of the neurotoxic $\mathrm{N}$-methyl-d-aspartate (NMDA) glutamate agonist quinolinic acid and 3-hydroxykynurenine subsequently improving oxidative stress and contributes to neurodegeneration which characterize MDD. (Leonard BE.) Whereas antidepressants attenuate some of the endocrine and immune changes caused by inflammation, not all therapeutically effective antidepressants do so. This suggests that drugs which particularly target the immune, endocrine and neurotransmitter systems may be more compelling than ordinary antidepressants which opens a new avenue for CBD. The blood levels of sIL-2R, TNF-a and IL-6 have been found high in MDD has moreover been affirmed by a metaanalysis [11] Decreased degrees of interleukins in MDD such as beneficial IL 10 are also questioned. Elevated CRP and IL-6 were discovered in people with MDD, and MDD was related with higher CRP levels in MDD Similarly, higher CRP and IL-6 levels are related with psychological indications of MDD.

Inflammatory markers do decrease BDNF articulation yet in resistant MDD patients both IL-1ß plasma concentrations are increased making them as partial yet effective candidates to identifying MDD. It is obvious that cytokines induce behavioral effects by activating inflammatory signaling pathways in the brain, leading to the reduction of growth factors such as BDNF and the expression of these growth factors is affected by immune cells and the immune factors they secrete.

The treatment resistance is also a part of inflammation and several markers are unfortunately neglected in psychiatry. CBD seems eliminate proinflammatory cytokines such as interleukin-1, interleukin-6, and interferon (IFN) and NF-KB pathway all causing to trigger the proinflammatory genes. CBD up-regulates the STAT3 and restores homeostatic mechanisms. Altered BDNF function is involved in the structural changes and possibly impaired neurogenesis, neurotoxicity and neuroinflammation in the brain of MDD patients showing a significant relationship between failed treatments and (TNF), soluble TNF receptor 2 (sTNF-R2) and interleukin (IL)-6. Checking inflammatory markers and targeting inflammation or its downstream mediators via diverse treatment options is relevant for MDD patients.

CBD can reestablish cannabinoid/GABAergic signaling deficits in regions of the brain implicated in MDD. Cannabidiol enhances 5-HT and glutamate levels in prefrontal cortex. 5-HT1A receptor mediates cannabidiol-induced antidepressant-like impacts and increase of 5-HT/glutamate levels. One of the basic role of CBD is that it diminish inflammation within the central region of the brain decreases levels of proinflammatory cytokines within the hippocampus (TNF-a) and prefrontal cortex (IL-6). Inflammation and but moreover infection diminish BDNF gene expression and so CBD increases BDNF by diminishing inflammation within the brain with its anti- inflammatory activity which is exceptionally critical figure that standard medicines can do it only to a minimum degree [12] CBD plays a basic part in eliminating the inflammation as seen in serum/plasma inflammatory markers. The ECS has been shown both in vivo and in vitro to be included in directing the immune system through its immunomodulatory properties. CBD work by binding several subtypes of cannabinoid receptor within the brain and other organs. In expansion, the body produces ECs that moreover work through binding these receptors. Compounds that are chemically related to $\mathrm{CBD}$ have also been shown to function by binding other sorts of receptor, such as the NMDA (N-methyl-D-aspartate) receptor and the peroxisome-proliferativeactivated receptor-(PPAR-), or by affecting other cellular components, such as lipid rafts.

$\mathrm{CBD}$ and related compounds have been shown to either suppress or increase the production of pro-inflammatory cytokines such as tumornecrosis factor (TNF-alpha), interleukin-1ß (IL-1ß) and IL-6 in both patients and animal models, indicating that these drugs can modulate pro-inflammatory mediators. Depending on the model system, the effects of these drugs do not always depend on their interaction with cannabinoid receptors. CBD disposits the immune response away from $\mathrm{T}$ helper 1 (TH1)-cell responses, by mechanisms that involve cannabinoid receptors. It is possible that signaling through these receptors, expressed by $\mathrm{T}$ cells, B cells or antigen-presenting cells, suppresses the expression of TH1-cell-promoting cytokines and increases the expression of TH2-cell-promoting cytokines. CBD and regulate some of the inflammatory aspects of brain injury, through both cannabinoid-receptor-mediated and non-cannabinoid-receptormediated mechanisms. It is conceivable that $\mathrm{eCB}$ decrease brain edema and other aspects of neuroinflammation by inhibiting NMDA receptors, by functioning as antioxidants and by decreasing the levels of pro-inflammatory cytokines within the brain. CBD suppress inflammatory reaction and in this way attenuate disease symptoms. This property of CBD is intervened through numerous pathways such as induction of apoptosis in activated resistant cells, concealment of cytokines and chemokines at inflammatory sites and upregulation of FoxP3+ regulatory $\mathrm{T}$ cells. Increased inflammation not only happens in MDD but also in numerous other psychiatric illnesses including bipolar disorder, anxiety disorders, identity disorders, and schizophrenia. These data propose that inflammation is transdiagnostic in nature, happening in subpopulations of patients inside a number of psychiatric disorders and so making this hypothesis not a sole candidate for clarifying completely the MDD.

\section{Neuroplasticity Theory of MDD}

The recently emerged neuroplasticity theory of MDD is additionally an approach to clarify the pathogenesis of MDD. Neuroplasticity is considered to be one of the imperative characteristics of the nervous tissue which shows in reversible changes which is called functional plasticity. It appears to modulate the expression of genotype into phenotype by persistent adjustment and thus bring around long-lasting impacts which is fundamental for MDD. 
This hypothesis sets that depressive indications emerge from the impaired neuroplasticity, which can be initiated by numerous risky components, including neurotransmitter imbalance and insufficient BDNF. MDD patients not only show impeded neurogenesis but also present disturbed neuronal growth, decreased synaptic plasticity, impaired myelin function, and a decrease in total neuroplasticity. CBD's impacts of plasticity can cause either positive or negative changes amid developmental plasticity, receptive plasticity, adaptational plasticity, and amid primary or auxiliary recuperation of damaged neuronal circuits, reparation plasticity. CBD in conjunction with neuroplastic mechanisms help repair and reestablish the neuronal circuitry damaged by MDD.

As CBD is appeared to upgrade BDNF expression it indirectly leads to form new connections by enacting the BDNF-TrkB signaling pathway. Since CBD's affect is blocked when BDNF signaling is hindered within the brain, CBD starts to set up quick neurochemical and neuroplastic effects in limbic brain regions favoring stress coping strategies and resilience to MDD improvement. This theory is promising in all sense yet as neuroplasticity evolves with other components of abovementioned theories it is also not capable to fully explain MDD by isolating other neurological and psychiatric disorders and diseases.

\section{Discussion}

CBD has a complex pharmacology and it interacts with multiple neurotransmitter systems involved in MDD such as serotonergic, glutamatergic, and endocannabinoid systems. CBD induces cellular and molecular changes in brain regions related to MDD neurobiology, such as increased BDNF levels and synaptogenesis in the medial prefrontal cortex, as well as it increases neurogenesis in the hippocampus. Although all factors related to the abovementioned theories are considered playing roles in MDD none of them could be accepted as sole candidates and there is no "unified theory" to explain the cause the MDD but almost all studies underline the effects of CBD and indicate CBD as a safe option for applying as alone or in combination with conventional drugs as an adjuvant medication in MDD. CBD responds to several receptors known to regulate fear and anxiety-related behaviors, specifically the cannabinoid type 1 receptor (CB1R), the 5-HT1A receptor, and the transient receptor potential (TRP) vanilloid type 1 (TRPV1) receptor. In addition, CBD may also regulate, directly or indirectly, the peroxisome proliferatoractivated receptor- $\gamma$, the orphan G-protein-coupled receptor 55, the equilibrative nucleoside transporter, the adenosine transporter, additional TRP channels, and glycine receptors. Within this respect CBD regulates the needed catecholamines, anti-inflammatory markers and homeostasis too. Despite the fact that reviews have exhibited the quieting, calming, loosening up and anti-inflammatory impacts of CBD, clinical information from real and human cases is insignificant. Endocannabinoid system's relation with MDD and other points should be explored in extensive studies. When tested over a wide range of doses in further studies, the anxiolytic effects of CBD presented a bell-shaped dose-response curve, with anxiolytic effects observed at moderate but not higher doses. Preclinical evidence conclusively demonstrates CBD's efficacy in reducing anxiety behaviors in MDD. Current preclinical and human findings mostly involve acute CBD dosing in healthy subjects, so further studies are required to establish whether chronic dosing of CBD has similar effects in relevant clinical populations.

\section{Conclusion}

The principle finding of studies and practices is that $\mathrm{CBD}$ alongside conventional prescriptions and enhancements can be a successful compound to diminish Major Depressive Disease other related issues as showed in a critical or analytical summing up especially of a medical case history of several patients. Further investigation should be directed to decide the permanency of patient's certain practices and to what extent they should keep taking the CBD. As CBD interacts with BDNF in a positive manner it is highly possible a resilience to MDD symptoms and maybe a long lasting healing. Since CBD possesses also anti-inflammatory properties it directly hits the immune system anomalies so contributing also to improvements. Although human trials on CBD and ECS with respect to MDD is still not satisfying observational data indicates a need to increase them. It is a solid fact that $\mathrm{CBD}$ is used among patients and therefore physicians should not disregard patients' interest in cannabidiol as treatment option and continue to enlighten both patients and themselves about alternative therapies with the assistance and guidance of translational medicine experts. Combinations of CBD and conventional medications are of utmost importance and needed as necessary as patient's unique medical conditions such as endocrinology, cardiology, gastroenterology etc. The ECS' vital role in MDD still needs extensive studies. There are basic and emerging hypotheses about how MDD develops but none of them suffice to clarify this problematic to a satisfying degree. This phenomenon is open to debate and possibly it is time to consider that there's no academic need for persistency for each one and a translational approach is the solution which is of utmost importance. A scientific and sound need for all of these theories ought to be utilized to illuminate different perspectives of MDD and so possibly to offer actually a combined and unified theory which is the one intertwined and open to future investigations. Extensive literature search indicates that ever since the 1970s, a number of scientific articles studying the potential therapeutic impacts of CBD in different animal models of neuropsychiatric disorders and a few human clinical trials have been published. Since then knowledge about the new impacts of ECS and CBD and the components of action of this compounds have emerged. New questions showed up with respect to the properties of $\mathrm{CBD}$ and its safety and exact dosage ranges for each disorder. Hence, more controlled randomized, placebo-controlled clinical trials with larger populations will bring critical answers and support the interpretation support the translation of them to clinical settings.

\section{Acknowledgement}

This Review Intends To Put Translational Medicine to the Attention of Physicians

\section{References}

1. Herkenham M, Lynn A B, Johnson M.R, Melvin LS, De Costa (1991) Characterization and localization of cannabinoid receptors in rat brain: a quantitative in vitro autoradiographic study. Journal of Neuroscience 11: 563-583.

2. Vinod KY, Hungund BL (2006) Role of the endocannabinoid system in depression and suicide. Trends Pharmacol Sci 27: 539-545.

3. Russo EB, Burnett A, Hall B, Parker KK (2005) Agonistic properties of cannabidiol at, 5-HT1a receptors. Neurochem Res 30: 1037-1043. 
4. Linge $\mathrm{R}$ (2016) Cannabidiol induces rapid-acting antidepressant-like effects and enhances cortical 5-HT/glutamate neurotransmission: role of 5-HT1A receptors. Neuropharmacology 103: 16-26.

5. Fogaça MV, Reis FM, Campos AC, Guimaraes FS (2014) Effects of intra-prelimbic prefrontal cortex injection of cannabidiol on anxiety-like behavior: involvement of 5HT1A receptors and previous stressful experience. Eur Neuropsychopharmacol 24: 410-419.

6. Giacomo V, Chiavaroli A, Orlando G, Cataldi A, Rapino M, et al. (2020) Neuroprotective and Neuromodulatory Effects Induced by Cannabidiol and Cannabigerol in Rat Hypo-E22 cells and Isolated Hypothalamus. Antioxidants 9: 71 .

7. Campos AC, Fogaça Mv, Scarante FF, Joca S, Sales AJ, et al. (2017) Plas tic and Neuroprotective Mechanisms Involved in the Therapeutic Effects of Cannabidiol in Psychiatric Disorders. Frontiers in pharmacology 8: 269.

8. Niederhoffer N, Hansen HH, Fernandez Ruiz JJ, Szabo B (2001) Effects of cannabinoids on adrenaline release from adrenal medullary cells. British journal of pharmacology, 134: 1319-1327.
9. Barden N (2004) Implication of the hypothalamic-pituitary-adrenal axis in the physiopathology of depression. J Psychiatry Neurosci 29:185-193.9

10. Leonard BE (2018) Inflammation and depression: a causal or coincidental link to the pathophysiology? Acta Neuropsychiatr 30: 1-16.

11. Liu Y, Ho RC, Mak A (2012) Interleukin (IL)-6, tumour necrosis factor alpha (TNF- $\alpha$ ) and soluble interleukin-2 receptors (sIL-2R) are elevated in patients with major depressive disorder: a meta-analysis and meta-regression. J Affect Disord 139: 230-239.

12. Calabrese F, Rossetti AC, Racagni G, Gass P, Riva MA, et al. (2014) Brain-derived neurotrophic factor: a bridge between inflammation and neuroplasticity. Front Cell Neurosci. 


\section{If}

Advances In Industrial Biotechnology | ISSN: 2639-5665

Advances In Microbiology Research | ISSN: 2689-694X

Archives Of Surgery And Surgical Education | ISSN: 2689-3126

Archives Of Urology

Archives Of Zoological Studies | ISSN: 2640-7779

Current Trends Medical And Biological Engineering

International Journal Of Case Reports And Therapeutic Studies | ISSN: 2689-310X

Journal Of Addiction \& Addictive Disorders | ISSN: 2578-7276

Journal Of Agronomy \& Agricultural Science | ISSN: 2689-8292

Journal Of AIDS Clinical Research \& STDs | ISSN: 2572-7370

Journal Of Alcoholism Drug Abuse \& Substance Dependence | ISSN: 2572-9594

Journal Of Allergy Disorders \& Therapy | ISSN: 2470-749X

Journal Of Alternative Complementary \& Integrative Medicine | ISSN: 2470-7562

Journal Of Alzheimers \& Neurodegenerative Diseases | ISSN: 2572-9608

Journal Of Anesthesia \& Clinical Care | ISSN: 2378-8879

Journal Of Angiology \& Vascular Surgery | ISSN: 2572-7397

Journal Of Animal Research \& Veterinary Science | ISSN: 2639-3751

Journal Of Aquaculture \& Fisheries | ISSN: 2576-5523

Journal Of Atmospheric \& Earth Sciences | ISSN: 2689-8780

Journal Of Biotech Research \& Biochemistry

Journal Of Brain \& Neuroscience Research

Journal Of Cancer Biology \& Treatment | ISSN: 2470-7546

Journal Of Cardiology Study \& Research | ISSN: 2640-768X

Journal Of Cell Biology \& Cell Metabolism | ISSN: 2381-1943

Journal Of Clinical Dermatology \& Therapy | ISSN: 2378-8771

Journal Of Clinical Immunology \& Immunotherapy | ISSN: 2378-8844

Journal Of Clinical Studies \& Medical Case Reports | ISSN: 2378-8801

Journal Of Community Medicine \& Public Health Care | ISSN: 2381-1978

Journal Of Cytology \& Tissue Biology | ISSN: 2378-9107

Journal Of Dairy Research \& Technology | ISSN: 2688-9315

Journal Of Dentistry Oral Health \& Cosmesis | ISSN: 2473-6783

Journal Of Diabetes \& Metabolic Disorders | ISSN: 2381-201X

Journal Of Emergency Medicine Trauma \& Surgical Care | ISSN: 2378-8798

Journal Of Environmental Science Current Research | ISSN: 2643-5020

Journal Of Food Science \& Nutrition | ISSN: 2470-1076

Journal Of Forensic Legal \& Investigative Sciences | ISSN: 2473-733X

Journal Of Gastroenterology \& Hepatology Research | ISSN: 2574-2566
Journal Of Genetics \& Genomic Sciences | ISSN: 2574-2485

Journal Of Gerontology \& Geriatric Medicine | ISSN: 2381-8662

Journal Of Hematology Blood Transfusion \& Disorders | ISSN: 2572-2999

Journal Of Hospice \& Palliative Medical Care

Journal Of Human Endocrinology | ISSN: 2572-9640

Journal Of Infectious \& Non Infectious Diseases | ISSN: 2381-8654

Journal Of Internal Medicine \& Primary Healthcare | ISSN: 2574-2493

Journal Of Light \& Laser Current Trends

Journal Of Medicine Study \& Research | ISSN: 2639-5657

Journal Of Modern Chemical Sciences

Journal Of Nanotechnology Nanomedicine \& Nanobiotechnology | ISSN: 2381-2044

Journal Of Neonatology \& Clinical Pediatrics | ISSN: 2378-878X

Journal Of Nephrology \& Renal Therapy | ISSN: 2473-7313

Journal Of Non Invasive Vascular Investigation | ISSN: 2572-7400

Journal Of Nuclear Medicine Radiology \& Radiation Therapy | ISSN: 2572-7419

Journal Of Obesity \& Weight Loss | ISSN: 2473-7372

Journal Of Ophthalmology \& Clinical Research | ISSN: 2378-8887

Journal Of Orthopedic Research \& Physiotherapy | ISSN: 2381-2052

Journal Of Otolaryngology Head \& Neck Surgery | ISSN: 2573-010X

Journal Of Pathology Clinical \& Medical Research

Journal Of Pharmacology Pharmaceutics \& Pharmacovigilance | ISSN: 2639-5649

Journal Of Physical Medicine Rehabilitation \& Disabilities | ISSN: 2381-8670

Journal Of Plant Science Current Research | ISSN: 2639-3743

Journal Of Practical \& Professional Nursing | ISSN: 2639-5681

Journal Of Protein Research \& Bioinformatics

Journal Of Psychiatry Depression \& Anxiety | ISSN: 2573-0150

Journal Of Pulmonary Medicine \& Respiratory Research | ISSN: 2573-0177

Journal Of Reproductive Medicine Gynaecology \& Obstetrics | ISSN: 2574-2574

Journal Of Stem Cells Research Development \& Therapy | ISSN: 2381-2060

Journal Of Surgery Current Trends \& Innovations | ISSN: 2578-7284

Journal Of Toxicology Current Research | ISSN: 2639-3735

Journal Of Translational Science And Research

Journal Of Vaccines Research \& Vaccination | ISSN: 2573-0193

Journal Of Virology \& Antivirals

Sports Medicine And Injury Care Journal | ISSN: 2689-8829

Trends In Anatomy \& Physiology | ISSN: 2640-7752

Submit Your Manuscript: https://www.heraldopenaccess.us/submit-manuscript 